\title{
Endovascular Treatment of Symptomatic Vertebral Artery Dissecting Aneurysms
}

\author{
Jinsol Han', Dong-Jun Lim', Sung-Kon Ha', Jong-ll Choi', Sung-Won Jin', Se-Hoon Kim' \\ 'Department of Neurosurgery, Korea University Ansan Hospital, Ansan, Korea \\ ${ }^{2}$ Department of Neurosurgery, Hallym University Kangnam Sacred Heart Hospital, Seoul, Korea
}

Objective : Vertebral artery dissecting aneurysms (VADAs) are rare and many debates are present about treatment options. We review types and efficacy of our endovascular treatments and establish a safe endovascular therapeutic strategy regard to the angio-architecture of VADAs.

Materials and Methods : Between July 2008 and October 2015, we treated 22 patients with symptomatic VADAs. Fifteen patients presented with subarachnoid hemorrhage from the ruptured VADAs, digital subtraction angiography and magnetic resonance image confirmed the diagnosis and endovascular treatments were followed as their angio-architecture.

Results : Clinical results were good in 13 patients (86.7\%), and there were no technical problems during endovascular procedures. The other 2 patients with poor prognosis showed severe neurological deficits at the initial evaluation. Among the three different endovascular treatments, there were no radiologic cure in one patient with stent insertion alone, but the patient had no significant clinical symptoms either.

Conclusion : Endovascular treatments are safe and effective treatment option for managing VADAs and can be the first treatment of choice for most patients. To select proper endovascular treatment according to the angio-architecture of VADAs can reduce the risk of the treatment.

Keywords Vertebral artery, Dissecting aneurysms, Embolization

\author{
J Cerebrovasc Endovasc Neurosurg. \\ 2016 September; 18(3):201-207 \\ Received : 25 April 2016 \\ Revised : 29 August 2016 \\ Accepted : 6 September 2016
}

\begin{abstract}
Correspondence to Dong-Jun Lim Department of Neurosurgery, Ansan Hospital, Korea University Medical Center, 123 Jeokgeumro Danwon-gu, Ansan 15355, Korea

Tel : 82-31-412-5053

Fax : 82-31-412-5054

E-mail : djlim@korea.ac.kr

ORCID : http://orcid.org/0000-0002-2396-692X
\end{abstract}

The 56th Annual Meeting of the Korean Neurosurgical Society; e-Poster

\section{INTRODUCTION}

Vertebral artery dissecting aneurysms (VADAs) frequently occur in the extradural portion of the vertebral artery (VA) and commonly present with headache, neck pain or neurological deficits caused by ischemia. ${ }^{3{ }^{39)}}$ Those aneurysms are an unusual but significant cause of non-traumatic subarachnoid hemorrhage $(\mathrm{SAH})$, representing $3 \%$ of all intracranial aneurysm. ${ }^{5613)}$ Because the risk of re-rupture is high in cases presenting with $\mathrm{SAH}$, urgent treatment is needed. ${ }^{2779)}$
However the treatment options for VADAs are limited because of their angio-architecture. Total occlusion of the parent artery has become a broadly established approach for treating VADAs, but also it brings a probable risk of ischemic results in the posterior circulation territories when the VA is abruptly occluded, mostly in patients who have a hypoplastic contralateral $\mathrm{VA}^{1{ }^{15)(12)}}$

In this study, we report our experience with endovascular treatment of 15 patients presented with $\mathrm{SAH}$ who had symptomatic VADAs and establish a safe endovascular therapeutic strategy regard to the an- 
gio-architecture of VADAs.

\section{MATERIALS AND METHODS}

\section{Patients}

This is a retrospective study of 22 consecutive patients with VADAs presenting variable clinical features. Fifteen patients among them showed specific symptoms in regard to SAH and they were treated with endovascular strategies. A total of 7 males and 8 females were included in the study group, with ages ranging from 36 to 68 years (mean age, 48.6 years). Hunt Hess scale was used to evaluate the patients at admission, and modified Rankin Score (mRS) was used to evaluate patients not only at admission (Initial mRS) but also at 3 months after procedure (Post-procedure mRS). However, there were 2 patients who were expired or transferred out, so that the last mRS of those patients were included.

\section{Angio-architecture and endovascular treatment}

Diagnosis were confirmed using digital subtraction angiography (DSA) and magnetic resonance image
(MRI), if one or both of the following qualifications were encountered: 1) The dissecting aneurysm was related to angio-architectures defined below and 2) MRI confirmed a false lumen relating the parent artery and excluded luminal stenosis or fusiform transformation due to atherosclerosis.

The main angio-architecture in this study were divided into the following 4 groups: "pearl and string sign" (corresponding to a fusiform dilation associated with proximal or distal narrowing; 9 dissections), "string sign" (corresponding to an isolated irregular narrowing; 1 dissection), "fusiform dilation" (3 dissections), and "saccular aneurysm" (made by dissection with wide neck portion; 2 dissections).

DSAs were also assessed another important angio-architectures around them. The first was the dominancy of the VA, and the second was the relation between the posterior inferior cerebellar artery (PICA) and the dissecting aneurysm. Of the 15 VADAs, 5 cases were at the dominant VA, and 7 cases were at the non-dominant VA. The rest 3 cases showed co-dominant VA. In the relation with the PICA, 12 cases were distal to the PICA and 3 cases were proximal to the

Table 1. Clinical characteristics and angio-architectures of the patients

\begin{tabular}{cccccccc}
\hline No. & Age / Sex & HHS & Initial mRS* $^{*}$ & Characteristics of DSA & VA dominancy & Relation with PICA & Results of BTO \\
\hline 1 & $43 / \mathrm{M}$ & 1 & 1 & Pearl and string sign & Non-dominant & Distal to the PICA & Not performed \\
2 & $36 / \mathrm{F}$ & 2 & 1 & Pearl and string sign & Non-dominant & Distal to the PICA & Not performed \\
3 & $39 / \mathrm{F}$ & 2 & 1 & Pearl and string sign & Co-dominant & Distal to the PICA & Not performed \\
4 & $52 / \mathrm{F}$ & 2 & 1 & Pearl and string sign & Non-dominant & Distal to the PICA & Not performed \\
5 & $39 / \mathrm{F}$ & 2 & 1 & Pearl and string sign & Co-dominant & Distal to the PICA & Not performed \\
6 & $47 / \mathrm{F}$ & 1 & 0 & String sign & Non-dominant & Distal to the PICA & Not performed \\
7 & $51 / \mathrm{F}$ & 2 & 1 & Fusiform dilation & Non-dominant & Distal to the PICA & Not performed \\
8 & $55 / \mathrm{M}$ & 2 & 1 & Fusiform dilation & Dominant & Distal to the PICA & Negative \\
9 & $45 / \mathrm{M}$ & 2 & 1 & Fusiform dilation & Dominant & Distal to the PICA & Positive \\
10 & $51 / \mathrm{M}$ & 3 & 3 & Pearl and string sign & Non-dominant & Proximal to the PICA & Negative \\
11 & $58 / \mathrm{M}$ & 1 & 0 & Pearl and string sign & Non-dominant & Proximal to the PICA & Negative \\
12 & $68 / \mathrm{F}$ & 4 & 5 & Saccular aneurysm & Dominant & Distal to the PICA & Negative \\
13 & $51 / \mathrm{M}$ & 2 & 1 & Saccular aneurysm & Dominant & Proximal to the PICA & Negative \\
14 & $52 / \mathrm{F}$ & 2 & 1 & Pearl and string sign & Non-dominant & Distal to the PICA & Not performed \\
15 & $43 / \mathrm{M}$ & 2 & 1 & Pearl and string sign & Co-dominant & Distal to the PICA & Not performed \\
\hline
\end{tabular}

HHS = hunt hess scale; mRS = modified Rankin scale; DSA = digital subtraction angiography; VA = vertebral artery; PICA = posterior inferior cerebellar artery; BTO = Balloon test occlusion.

*The mRS evaluated at admission.

${ }^{\dagger}$ The main angio-architecture which is a qualification for diagnosis of dissecting aneurysms in DSA 
PICA. If the VADA is located at the dominant VA or proximal to the PICA, additional balloon test occlusion (BTO) was performed before selecting the treatment option. In considering those angio-architectures and results of BTO, treatment technics were divided into the following 3 technics: VA coil trapping, stent assisted coil embolization, and stent insertion alone. The summary of clinical characteristics of patients include Initial mRS, angio-architectures, and results of BTO are presented in Table 1.

\section{RESULTS}

In the 3 different treatment technics, 12 patients
$(80 \%)$ were treated with VA coil trapping (Fig. 1), 2 patients $(13 \%)$ with stent assisted coil embolization (Fig. 2), and 1 (6\%) patient with stent insertion alone (Fig. 3). There were no procedure-related complications such as extravasation of dye, distal embolic events, or coil migration.

In regard to the angio-architecture, 11 of 15 patients (73\%) showed the lesion was located at the non-dominant side or co-dominant side of the VA and 9 lesions among them were located distal to the PICA. VA coil trapping was performed for those 9 cases, the angiographic result showed complete occlusion. For the rest of 2 patients whose lesion was located proximal to the PICA, BTO was followed and the flow of con-
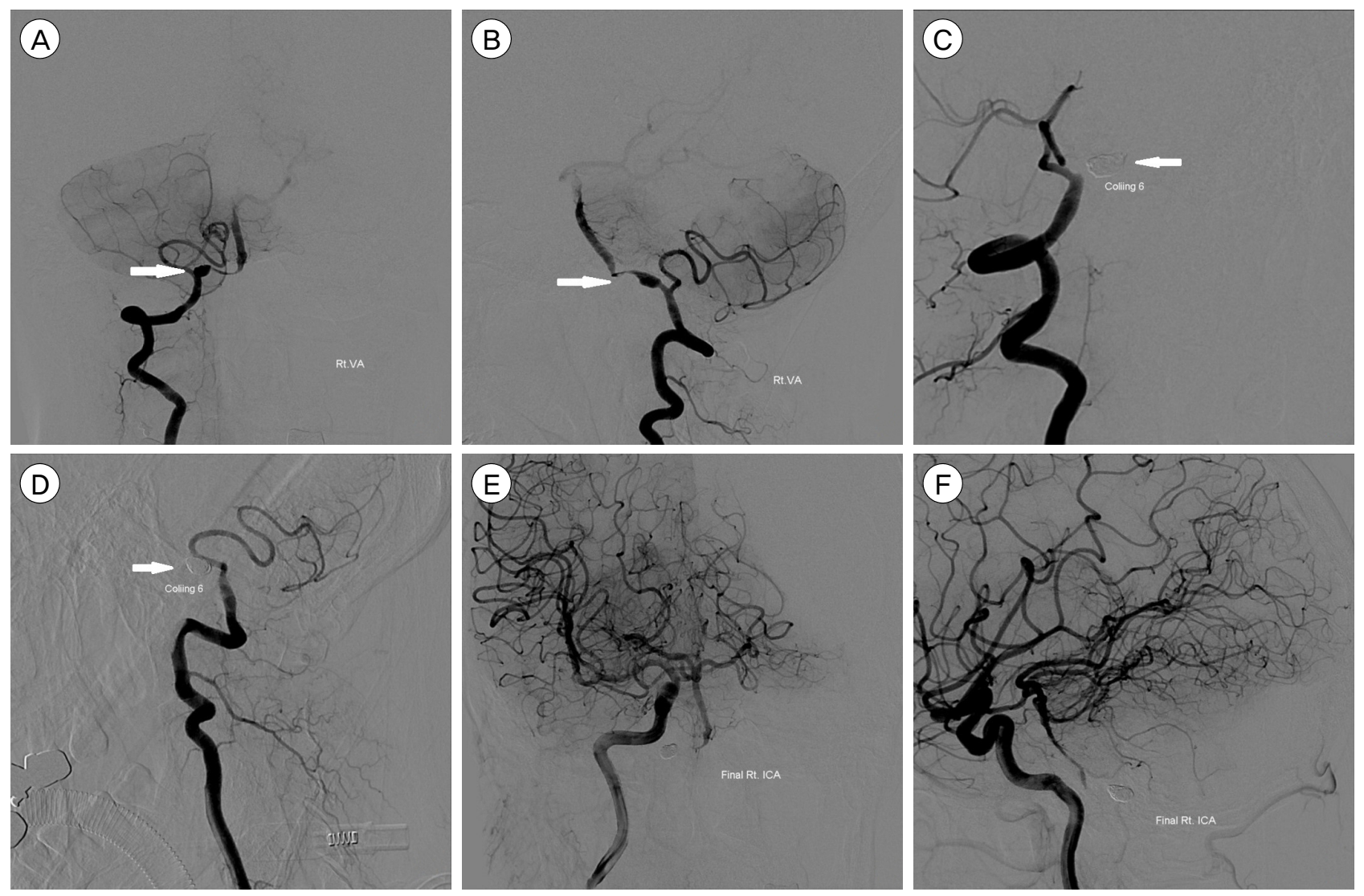

Fig. 1. Digital subtraction angiography (DSA) images of a 39-year-old woman presented with subarachnoid hemorrhage (SAH). The anterior-posterior (AP) and lateral view of the right vertebral angiogram shows a vertebral artery dissecting aneurysm (VADA) presented with "pearl and string sign", which is located at the distal to the origin of the posterior inferior cerebellar artery (PICA) (A, B; white arrow). Vertebral artery coil trapping technic was performed, and a total of 6 detachable coils, $37 \mathrm{~cm}$ in length, were placed into the dissecting segment and proximal parent artery, resulting in complete occlusion of the dissecting aneurysm with preservation of PICA flow (C, D; white arrow shows complete embolization of the VADA). The AP and lateral view of the right internal carotid angiogram shows sufficient collateral flow via right posterior communicating artery to the vertebrobasilar system without retrograde filling of the $\operatorname{VADA}(\mathrm{E}, \mathrm{F})$. 

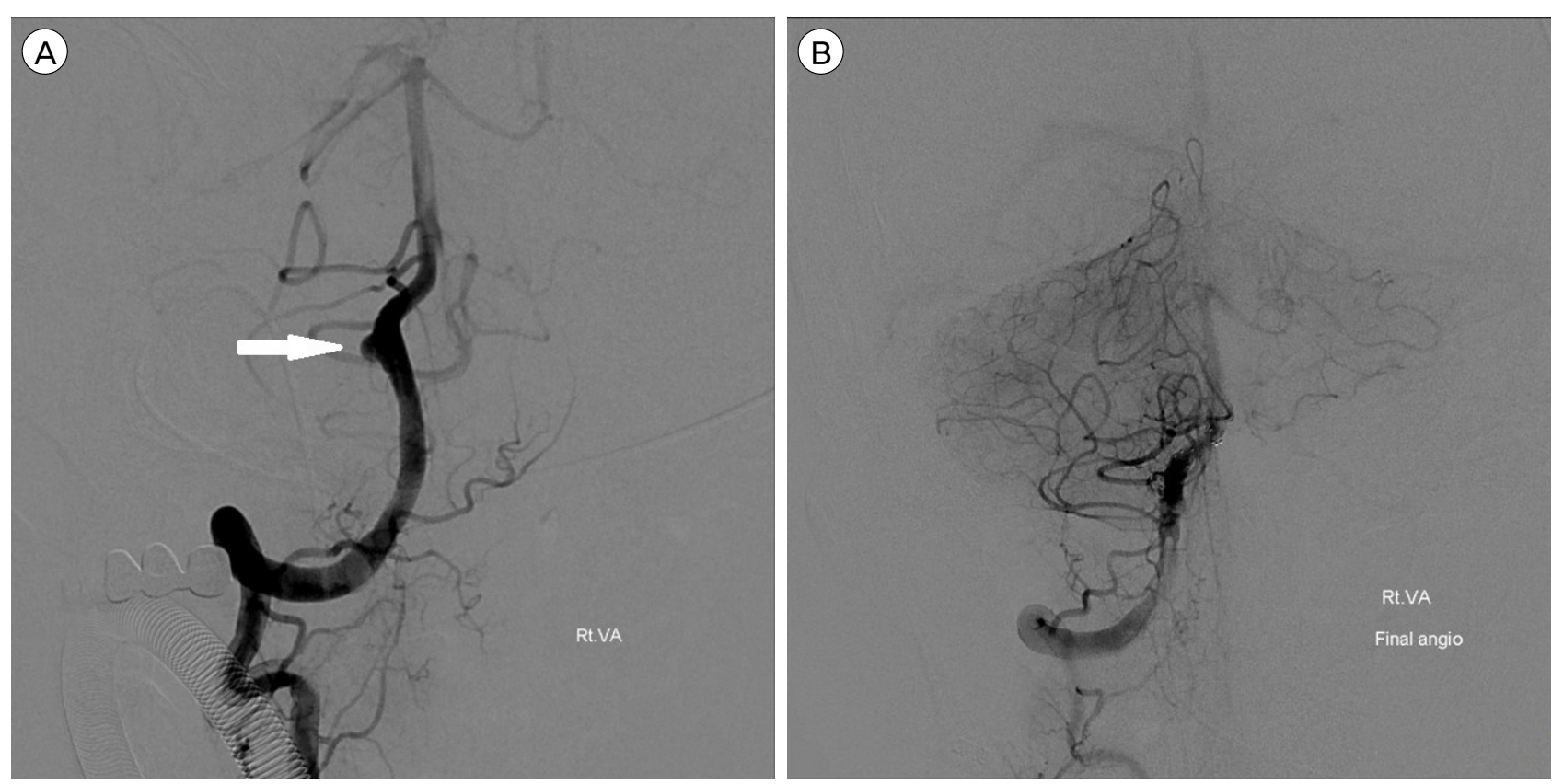

Fig. 2. DSA images of a 51-year-old man presented with $\mathrm{SAH}$ and $\mathrm{IVH}$. The lateral view of the right vertebral angiogram shows a VADA presented with "saccular aneurysm with wide neck" (A; white arrow). Double stent assisted coil embolization technic was performed, and a total of 4 detachable coils, $12 \mathrm{~cm}$ in length, were placed into the aneurysmal sac, resulting in near complete occlusion of the dissecting aneurysm with preservation of PICA and distal flow (B). DSA = Digital Subtraction Angiography; SAH = subarachnoid hemorrhage; IVH = intraventricular hemorrhage; VADA = vertebral artery dissecting aneurysm; PICA = posterior inferior cerebellar artery.

tralateral VA was filling enough to the ipsilateral PICA territory during the test, so that they also treated with VA coil trapping and the angiographic result showed complete occlusion.

The rest 4 patients $(27 \%)$ showed the lesion was at the dominant VA and additional BTO was followed. The result of 3 patients was negative and treatment options were divided as their angio-architectures; VA coil trapping for the lesion with "pearl and string sign" (1 patient), and stent-assisted coil embolization for the lesion with "saccular aneurysm" (2 patients). The result of BTO for the rest of 1 patient was positive and the patient showed "fusiform dilation" in the DSA, stent insertion alone technic was followed and
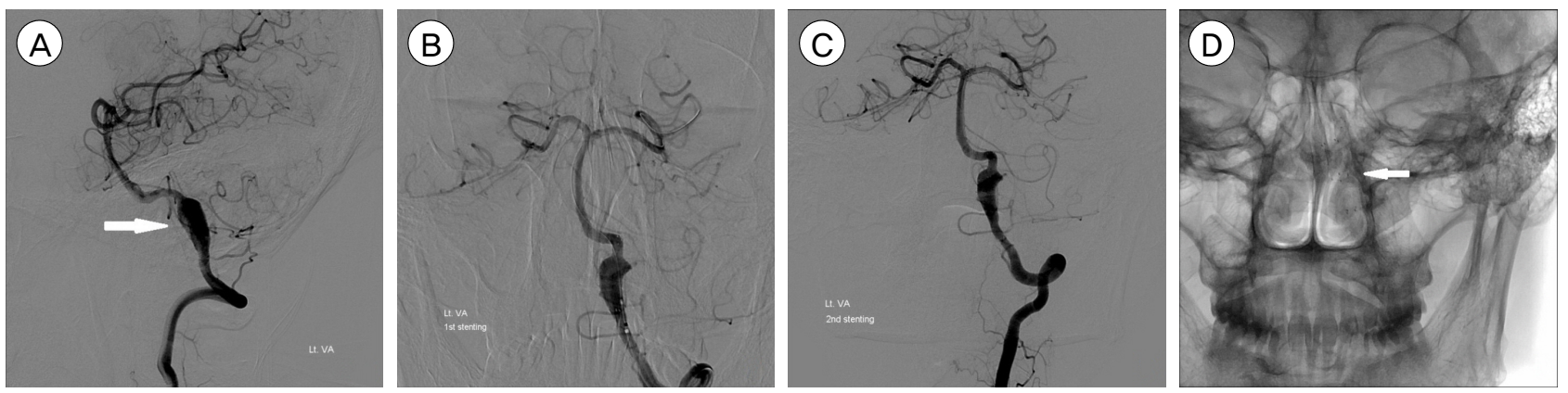

Fig. 3. DSA images of a 45-year-old man presented with SAH. The lateral view of the left vertebral angiogram shows a VADA presented with "fusiform dilation" (A; white arrow). Stent insertion alone technic was performed due to the balloon occlusion test was positive, and 2 stents were placed into the dissecting segment, resulting in incomplete occlusion of the dissecting aneurysm (B, C). The AP view of skull $x$-ray after stent insertion shows 2 stents are overlapped and well placed (D; white arrow). DSA = Digital Subtraction Angiography; SAH = subarachnoid hemorrhage; VADA = vertebral artery dissecting aneurysm. 
angiographic result was incomplete.

Thirteen patients $(86.7 \%)$ showed good clinical results in regard to Post-procedure mRS. Four patients $(26.7 \%)$ were evaluated as 0 point and the other 9 patients $(60 \%)$ were 1 point. The other 2 patients (13\%) were evaluated as 2 and 6 points respectively, the patient who expired 1 week after the endovascular treatment showed stuporous mentality at admission; Hunt Hess scale $=4$, and Initial $\mathrm{mRS}=5$ points. The summary of endovascular treatments and their outcomes are presented in Table 2.

\section{DISCUSSION}

VADA is a rare disease and the annual occurrence rate has been stated to be $1 / 100,000$ cases in the $\mathrm{USA}^{11)}$. It is common in the middle-aged, mostly $40-50$ years. Ruptured VADA patients presented with severe SAH usually show disastrous neurological results and a high occurrence rate of rebleeding. ${ }^{47) 8 \text { ) }}$ Mizutani et al. retrospectively analyzed 42 patients with re-bleeding triggered by the ruptured VADA and discovered that $40.5 \%$ of re-bleeding occurred within 24 hours and that 57.1\% occurred one week following the initial hemorrhage, ${ }^{7}$ so that after the diagnosis is confirmed, the treatment for ruptured VADA should be performed as soon as possible to prevent re-bleeding.

Furthermore, initial neurological status can be an important factor for predicting the prognosis. All patients in our study were presented with SAH and initially evaluated by Hunt Hess scale, 2 patients among them are score 3 and 4 respectively, and rest of 13 patients are score 1 or 2 . All the 15 patients were treated within 24 hours at the diagnosis, but the 2 patients with high Hunt Hess scale showed poor prognosis after the treatment. They were evaluated as 3 and 5 points for each of them at Initial mRS, 2 and 6 points respectively at Post-procedure mRS.

Endovascular treatments such as VA coil trapping, coil embolization of the aneurysmal sac with or without stent insertion, and stent insertion alone become the treatment of choice because VADAs do not have a real neck and conventional clipping does not manage the aneurysm successfully. ${ }^{10)}$ The angio-architecture of the vertebrobasilar system is the most im-

Table 2. The summary of endovascular treatments and their outcomes

\begin{tabular}{ccccc}
\hline No. & Treatment technics & Radiologic outcomes & $\begin{array}{c}\text { Clinical outcomes } \\
\text { (Post-procedure mRS })\end{array}$ & F/U months \\
\hline 1 & VA coil trapping & Complete & 0 & 32 \\
2 & VA coil trapping & Complete & 0 & 48 \\
3 & VA coil trapping & Complete & 1 & 26 \\
4 & VA coil trapping & Complete & 1 & 13 \\
5 & VA coil trapping & Complete & 1 & 86 \\
6 & VA coil trapping & Complete & 0 & 65 \\
7 & VA coil trapping & Complete & 1 & 5 (T/O) \\
8 & VA coil trapping & Complete & 1 & 57 \\
9 & Stent insertion alone & Incomplete & 1 & 20 \\
10 & VA coil trapping & Complete & 2 & 15 \\
11 & VA coil trapping & Complete & 0 & 24 \\
12 & Stent assisted coil embolization & Near complete & 6 & 0 \\
13 & Stent assisted coil embolization & Near complete & 1 & 8 \\
14 & VA coil trapping & Complete & 1 & 5 \\
\hline
\end{tabular}

$\mathrm{mRS}=$ modified Rankin Scale; F/U = Follow-up; $\mathrm{VA}=$ vertebral artery; $\mathrm{T} / \mathrm{O}=$ Transferred out.

*The mRS evaluated 3 months after procedure. If the patient was expired or transferred out before 3 months, the last mRS was included 
portant factor to select proper method of the endovascular treatments. We selected stent-assisted coil embolization in "saccular aneurysm" cases because the lesion could be well-localized, and we could preserve the flow of parent artery with making complete occlusion of the lesion. However coil embolization alone technic was impossible because of the aneurysmal sac had wide neck and the coil frame was unstable, so additional stent insertion was inevitable to prevent loosening of the coils.

We selected VA coil trapping as a first treatment of choice for the rest "pearl and string sign", "string sign" and "fusiform dilation" cases, because they could not be localized and we had to make complete occlusion both the lesion and the parent artery. In those cases, if the VADA was located at the non-dominant side or co-dominant side of the VA and distal to the PICA, we selected VA coil trapping as a treatment option without performing BTO. However, if the lesion was located at the dominant side of VA or proximal to the PICA, BTO should be performed before selecting the treatment option. If the result of BTO was negative, VA coil trapping could be still the first treatment of choice.

Six patients were performed BTO in our study and 5 patients of them showed negative results. VA coil trapping or stent-assisted coil embolization were performed as their angio-architecture. The other 1 patient showed positive result, and the angio-architecture of the patient was "fusiform dilation". We could apply limited treatment methods including a stent insertion alone technic or a flow diverter, so stent insertion alone technic was applied in our study as an alternative treatment option because of the lesion should not be occluded. The angiographic result was incomplete, but the clinical result of the patient was tolerable.

The limitations of our study are the small number of patients, the lack of comparative data for patients managed with surgical treatments, and the lack of long-term follow-up data. Especially, there was no mandatory short or long-term follow-up image studies after endovascular treatments, we could only estimate post-procedure mRS of patients. In the future, a randomized, multicenter trial with long-term follow up studies will be necessary to evaluate the safety and clinical efficacy of endovascular treatments for VADAs.

\section{CONCLUSION}

Endovascular treatments are safe and effective procedures for treating symptomatic VADAs, and the patients managed with endovascular treatments showed favorable clinical outcomes. To reduce the risk of the complication of endovascular treatments, it is important to analyze angio-architectures of the lesion in DSA and select proper procedure among the endovascular treatments.

\section{Disclosure}

The authors report no conflict of interest concerning the materials or methods used in this study or the findings specified in this paper.

\section{REFERENCES}

1. Andoh T, Shirakami S, Nakashima T, Nishimura Y, Sakai $\mathrm{N}$, Yamada $\mathrm{H}$, et al. Clinical analysis of a series of vertebral aneurysm cases. Neurosurgery. 1992 Dec;31(6):987-93.

2. Aoki N, Sakai T. Rebleeding from intracranial dissecting aneurysm in the vertebral artery. Stroke. 1990 Nov;21(11): 1628-31.

3. Arnold M, Bousser MG, Fahrni G, Fischer U, Georgiadis D, Gandjour J, et al. Vertebral artery dissection: presenting findings and predictors of outcome. Stroke. 2006 Oct;37(10):2499-503.

4. Kim MS. Endovascular coil trapping of a ruptured dissecting aneurysm of the vertebral artery using detachable coils and micro tornado ${ }^{\circledR}$ coils. J Cerebrovasc Endovasc Neurosurg. 2013 Jun;15(2):96-101.

5. Luo C-B, Chang CY, Teng MM, Chang FC. Endovascular treatment of ruptured vertebral dissecting aneurysms with electrodetachable coils. J Chin Med Assoc. 2005 Dec;68(12):578-84.

6. Lylyk P, Ceratto R, Hurvitz D, Basso A. Treatment of a vertebral dissecting aneurysm with stents and coils: technique and case report. Neurosurgery. 1998 Aug;43(2):385-8.

7. Mizutani T, Aruga T, Kirino T, Miki Y, Saito I, Tsuchida 
T. Recurrent subarachnoid hemorrhage from untreated ruptured vertebrobasilar dissecting aneurysms. Neurosurgery. 1995 May;36(5):905-11; discussion 912-3.

8. Nashimoto T, Komata T, Honma J, Yamashita S, Seki $Y$, Kurashima A, et al. Successful treatment of bilateral vertebral artery dissecting aneurysms with subarachnoid hemorrhage: report of three cases. J Stroke Cerebrovasc Dis. 2012 Jul;21(5):422-7.

9. Peluso JP, van Rooij WJ, Sluzewski M, Beute GN, Majoie CB. Endovascular treatment of symptomatic intradural vertebral dissecting aneurysms. AJNR Am J Neuroradiol. 2008 Jan;29(1):102-6.
10. Taha MM, Sakaida H, Asakura F, Maeda M, Toma N, Yamamoto A, et al. Endovascular management of vertebral artery dissecting aneurysms: review of 25 patients. Turk Neurosurg. 2010 Apr;20(2):126-35.

11. Wang Y, Zhao C, Hao X, Wang C, Wang Z. Endovascular interventional therapy and classification of vertebral artery dissecting aneurysms. Exp Ther Med. 2014 Nov;8(5):1409-15.

12. Yamaura A. Diagnosis and treatment of vertebral aneurysms. J Neurosurg. 1988 Sep;69(3):345-9.

13. Yamaura A, Watanabe Y, Saeki N. Dissecting aneurysms of the intracranial vertebral artery. J Neurosurg. 1990 Feb;72(2):183-8. 